\title{
Um estudo empírico sobre reações emocionais de usuários na interação com interfaces web pautadas na Gestalt
}

\author{
Diogo K. C. Nishikawa, Roberta P. Brandão, Vânia P. A. Neris \\ Departamento de Computação, Universidade Federal de São Carlos (UFSCar) \\ diogo.kcn@gmail.com, beta.enghaw@gmail.com, vania@dc.ufscar.br

\begin{abstract}
Resumo. Emoções influenciam decisões, comunicação com outras pessoas e com soluções web. As interfaces de usuário têm influência emocional sobre seu usuário e uma teoria para o design delas é a da Gestalt. Ainda é desconhecido sua influencia sobre as emoções durante uma interação. Este artigo realizou uma coleta de dados fisiológicos de voluntários durante o uso da solução web desenvolvida. Depois, classificou-se os dados de acordo com o Espaço Semântico de Scherer. Avaliou-se o impacto das leis sobre as emoções dos participantes. Os resultados sugeriram que as leis escolhidas mudaram de forma significativa uma pequena parcela dos voluntários.
\end{abstract}

\section{Introdução}

Nos últimos anos, as emoções passaram a ser um grande interesse da área de Interação Humano-Computador (IHC) e, por isso, pesquisas sobre o tema aumentaram, uma vez que pode ajudar as pessoas na tomada de decisões [Scherer 2005]. Elas afetam a atenção, a percepção, a memória, o comportamento, as decisões de compra e a aprendizagem [Beale and Peter 2008, Shami and et al. 2008].

Um bom design deve ser capaz de minimizar as emoções relacionadas à má usabilidade [Cristescu 2008]. Então, é preciso identificar novas maneiras de se avaliar os sistemas computacionais com atenção aos aspectos emocionais [Norman 2004, Piccolo et al. 2010]. Um dos modos com o qual os seres humanos conseguem distinguir se um determinado elemento, é bom ou ruim, é através de sua percepção visual. A Teoria da Gestalt [Köhler 1920, Wertherimer 1924], explica através de suas leis, como elementos individuais são visualmente agrupados em estruturas.

Pelo exposto, para avaliar o impacto emocional com a adoção de algumas leis em uma interface de usuário de uma solução web, este projeto coletou 210 amostras à partir de 15 voluntários por meio da captura de seus sinais fisiológicos e posterior classificação no Espaço Semântico de Scherer [Scherer 2005]. Cada participante realizou no total 14 tarefas. Em seguida, os dados foram refinados através da eliminação de possíveis ruídos, e, então, classificados pelo sistema de [Souza and Neris 2019]. Os resultados finais sugeriram uma diferença emocional com o uso das leis usadas para uma significativa parcela do grupo. Entretanto, uma parcela não apresentou mudanças no estado emocional.

Este estudo visa contribuir com discussões sobre a web social no que se refere a forma de apresentação de interfaces considerando o bem-estar dos usuários, mais especificamente, seu estado emocional. O restante deste trabalho encontra-se dividido em 4 seções: na seção 2 são abordados os conceitos fundamentais usados no projeto; na seção 3, os trabalhos relacionados; na seção 4, é explicado o estudo experimental; e, por fim, na seção 5, tem-se a conclusão e trabalhos futuros. 


\section{Conceitos Fundamentais}

\subsection{Emoções}

A definição de emoção existe em diversas áreas como psicologia, filosofia, neurociência e com as diferentes perspectivas, existem várias definições para o que seria emoção. Devido a falta de um consenso, Russell (2012) e Dixon (2012) recomendam a adoção da definição mais adequada com as características do trabalho em questão. Entre as definições, temos a de Pinto (2001), Damásio (2003), Braghirolli (2001), Norman (2004) e Scherer (2001).

Esta última destaca o modelo de processo de componente, no qual a emoção é conceituada como um episódio de mudança interrelacionada e sincronizada de todos, ou da maioria, dos subsistemas do organismo em resposta à avaliação de um evento de estímulo externo (resposta a eventos ou objetos do ambiente), ou interno (pensamentos sobre um evento ou objeto), como relevante para os principais interesses do organismo. A avaliação de estímulos tem por objetivo analisar objetos ou eventos com a finalidade de preparar no organismo uma reação apropriada [Scherer 2001].

Neste trabalho, adota-se a visão do modelo de componentes de Scherer e utiliza-se o Espaco Semântico [Scherer 1984], Figura 1, para a categorização de emoções.

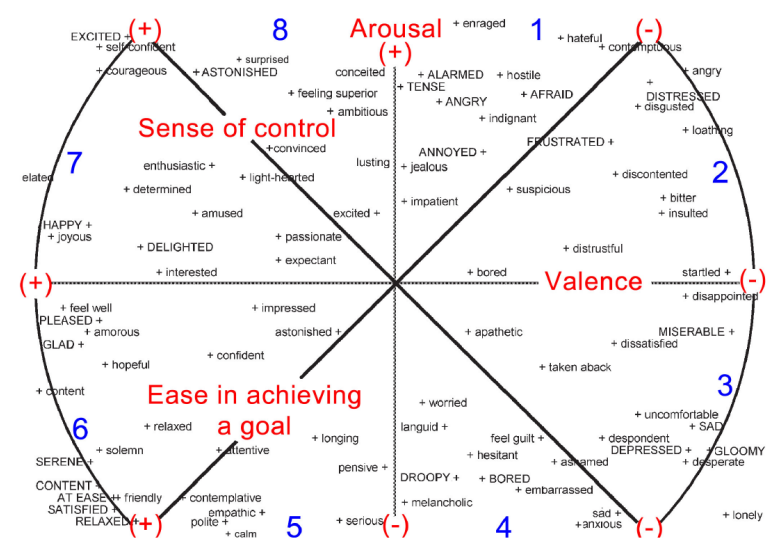

Figura 1. Estrutura do Espaço Emocional Semântico [Gonçalves 2016].

O Espaço Semântico é dividido em oito partes (oitantes), que representam quatro domínios emocionais: Valência, Excitação, Sentimento de controle e Facilidade de conclusão do objetivo. Cada um possui polos positivos e negativos. Através desse espaço é possível mapear o impacto que uma emoção provoca nos domínios da experiência emocional [Xavier et al. 2012].

As reações fisiológicas permitem avaliar o estado emocional, ao considerar que a emoção é motivada por evento ou objeto (estímulo) percebido e processado pelo sistema sensorial, e compartilhada com os demais sistemas do organismo. Indicadores de emoções incluem a resposta galvânica da pele, secura da boca, atividade cardíaca, atividade respiratória e atividade cerebral [Souza and Neris 2019].

Neste trabalho, foram usados os seguintes sinais fisiológicos: atividade cardíaca (a quantidade de vezes que o coração bate por minuto, medido pelo eletrocardiograma (ECG)), atividade cerebral (qual região cérebral está ativada em determinado estado emocional [Chang and et al. 2015]) e resposta galvânica da pele (a relação com a mudança 
de calor e condutividade, e o nível de excitação e situações de estresse ou frustração [Lichtenstein and et al. 2008, Mahlke and Mingue 2008] que pode ser mesurado pelo GSR).

\subsection{Gestalt}

A teoria da Gestalt é um conjunto de leis, ou princípios, que descrevem como os seres humano veem objetos à partir do agrupamento de objetos similares, reconhecimento de padrões e simplificação de imagens complexas em partes ou elementos individuais. Cada um desses elementos possuem suas próprias características e sua própria natureza, mas a natureza dos elementos individuais sozinha não informa como um grupo de elementos podem ser percebidos [Chang and Nesbitt 2006].

A partir de estudos dos princípios originais da Gestalt, formalizados por [Wertherimer 1924], foram identificados e propostos outros princípios [Palmer and Rock 1992, Palmer and Rock 1994]. Além disso, existem variações na teoria deixadas por diferentes psicólogos, como a extração de 114 leis, mas apenas uma pequena parte é aplicável para formas visuais [Boring 1942]. Esses princípios além de contribuirem para o entendimento da percepção visual humana, também trouxeram um entendimento à cerca da percepção auditiva humana no final dos anos 60.

Em um estudo feito por Chang e Nesbitt (2006), foram verificadas as 7 principais leis usadas, tanto em aplicações práticas e teóricas, para o sentido visual como também para o auditívo: lei do Fechamento, lei da proximidade, lei da continuação, lei da similaridade, lei da figura-fundo, lei do destino comum, lei do equilíbrio ou da simetria. As leis escolhidas para este experimento foram: da proximidade, da similaridade, do figura/fundo, do fechamento e a da região comum.

\section{Trabalhos Relacionados}

Três bases de dados foram consultadas: ACM Digital Library (ACM-DL), IEEE Xplore ${ }^{\circledR}$ Digital Library e ScienceDirect. Outras fontes também foram usadas, como a IEEE Transactions on Affective Computing, para os três últimos anos, e o site educacional Interaction Design Foundation. Efetuou-se também estudos sobre as leis da Gestalt em blogs indicados por este.

A revisão sistemática foi efetuada no período entre Abril e Maio do ano de 2019. Inicialmente, realizou-se as buscas com uma string genérica formada com as palavraschaves definidas e sinônimos, para averiguar o estado da arte, com o processo de refinação da string durante as pesquisas. A string geral final foi "(emotion OR affective OR gsr OR ecg OR eeg OR "user interface"OR interface) AND (gestalt)".

Para cada uma das bases de dados, mencionadas anteriormente, foram selecionadas as strings: "(emotion OR affective OR gsr OR ecg OR eeg OR "user interface" OR interface) AND (gestalt)"; "emotion OR affective OR gsr OR ecg OR eeg OR "user interface"OR interface) AND (gestalt)"; "(emotion OR affective) AND (gsr OR ecg OR eeg) AND ("user interface"OR interface) AND (gestalt)", respectivamente.

Como parte do protocolo foram definidos os seguintes critérios de inclusão: aplica leis da Gestalt para uma interface de usuário; utiliza sinais fisiológicos para inferir emoções; e descreve leis da Gestalt para design de interfaces de usuário. Os critérios 
de exclusão foram: não aplica leis da Gestalt para interface de usuário; não utiliza sinais fisiológicos para inferir emoções; não foi possível acessar o trabalho; e idioma que não seja português ou inglês.

Os temas encontrados nos trabalhos levantados abrangeram desde aplicações em sites de empresas de tecnologia (e.g. Apple, Microsoft) - visto em Sani e Shokooh (2016), e o uso das leis para a área da saúde como estudado em Chang, Dooley e Tuovinen (2002), que lida com saúde com sua ferramenta WoundCare. Além disso, pelo resultado da revisão sistemática foi possível verificar que não havia nenhum trabalho que relatasse o uso de sensores para a captura de sinais fisiológicos espeficicamente para análise de emoções causadas pela interação com uma interface de usuário pautada em algumas leis da Gestalt.

\section{Estudo Experimental}

\subsection{Planejamento}

Utilizou-se a abordagem de intragrupo para controle e conduta dos participantes cuja idade mínima deveria ser de 18 anos. Não houve critérios de exclusão e inclusão, uma vez que soluções web são usadas diariamente por grande parte da população.

Houve a participação de 15 voluntários (14 estudantes da área da Computação e um profissional da área da Pedagogia), com idades entre 18 e 29 anos e para cada um, atribuiu-se um ID. Todos familiarizados com tecnologia e sem experiência com a Teoria da Gestalt.

Com a aceitação da participação, explicou-se sobre o projeto e instruiu-se a leitura do Termo de Consentimento Livre e Esclarecido e a Autorização de Captação de Imagem, Som e Nome devido à gravação de voz (por causa do método Think aloud) e possível registro visual com foto. Além dos 2 questionários SAM, um após cada interação, e um questionário final de perfil.

Posteriormente, eram colocados os sensores: os eletrodos ECG no tronco do participante (colocados pelo próprio); GSR nos dedos das mãos e EEG em formato de uma tiara ou de um fone de ouvido na cabeça.

O experimento foi dividido em duas sessões de 10 a 15 minutos, aproximadamente. Na primeira, o voluntário interagia com umas das duas versões do site e para cada uma delas, executava 7 tarefas (iguais para as duas versões). Todas as tarefas, com suas descrições, podem ser vistas na Tabela 1 abaixo. Na Figura 2 b é possível ver uma comparação do uso da lei da Região Comum na ferramenta desenvolvida. Depois, era realizado uma pausa de até 5 minutos cujo objetivo era permitir que o participante repousasse entre os estímulos emocionais [Abadi and et al. 2015, Koelstra and et al. 2012, Subramanian and et al. 2016]. Feita a pausa, prosseguia-se para a interação da outra versão. A ordem de qual versão começar foi aleatória de modo a se obter no final uma distribuição quase justa dessa ordenação.

\subsection{Coleta}

Para a coleta por meio de sensores foi utilizado o sistema desenvolvido e construído por Souza e Neris (2019). Esse sistema é composto por sensores ECG, EEG e GSR de baixo custo, explicitados a seguir. Esses sensores possuem as taxas de acurácia de 99\%, 99\% e $78 \%$, respectivamente. 
Tabela 1. Tarefas e suas descrições

\begin{tabular}{|c|l|}
\hline Tarefa & Descrição \\
\hline 1 & $\begin{array}{l}\text { Contate a ONG e envie para ela uma mensagem com o seguinte texto: "Obri- } \\
\text { gado(a) pelos seus serviços e dedicação" }\end{array}$ \\
\hline 2 & $\begin{array}{l}\text { Leia alguns depoimentos - mínimo 4 e máximo 6 - dados por pessoas à ONG, } \\
\text { na seção "Doações" }\end{array}$ \\
\hline 3 & $\begin{array}{l}\text { Envie uma mensagem de interesse para se voluntariar. Utilize o botão "Juntar- } \\
\text { se" presente no rodapé do site }\end{array}$ \\
\hline 4 & $\begin{array}{l}\text { Leia as doações, em "Últimas Doações", presentes na página inicial (página } \\
\text { Home) }\end{array}$ \\
\hline 5 & $\begin{array}{l}\text { Procure um gato, na seção "Gatos", e entre em contato para adotá-lo. Utilize } \\
\text { o botão "Adotar" presente no rodapé do site }\end{array}$ \\
\hline 6 & Leia o primeiro parágrafo de "Um Pouco Sobre Nós", na seção "Sobre" \\
\hline 7 & Leia sobre a fundadora que trabalha no marketing, na seção "Sobre" \\
\hline
\end{tabular}

Para a coleta da resposta galvânica (GSR) foi feita com dois anéis elásticos colocados nos dedos (modelo considerado adequado por Boucsein (2012)), com uso do sensor Grove - GSR Sensor ${ }^{1}$ junto com o Arduino Leonardo ${ }^{2}$. Para a atividade cerebral (EEG), usou-se o sensor NeuroSky MindWave Headset. Os sinais observados foram: beta, alfa, teta e delta. Estes foram recebidos por interface de comunicação USB. Já para a atividade cárdica (ECG), o sensor ECG Heart Monitor AD823214, conectado ao Arduino Leonardo, e contém 3 eletrodos que são posicionados na região do tronco de uma pessoa.

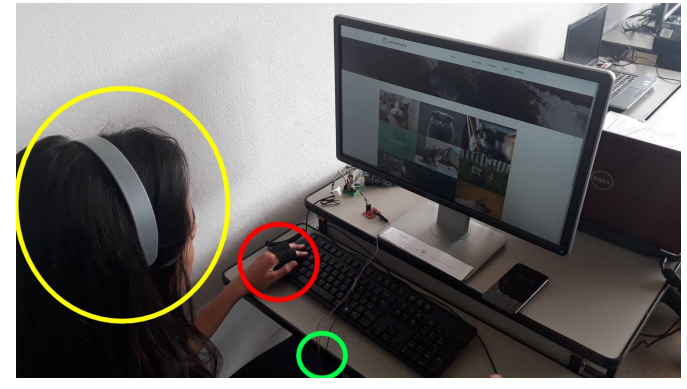

(a) Participante com os sensores.
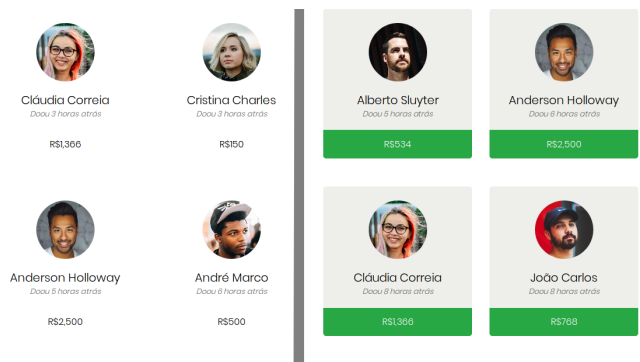

(b) Lei de Região Comum (Elaborada pelo autor).

Figura 2. Participante usando os três sensores e um exemplo comparativo de uma lei em um elemento de interface.

É possível visualizar na Figura 2a um dos voluntários interagindo com uma das versões do site enquanto está com os equipamentos sobre o corpo. Círculo amarelo indica o sensor EEG; o círculo vermelho, o sensor GSR em dois de seus dedos da mão esquerda; e o círculo verde, o sensor ECG.

\subsection{Análise}

Pela análise dos dados coletados e classificados, observou-se mudanças no estado emocional (mudança de para um conjunto de usuários em cada uma das 7 tarefas. As mudanças

\footnotetext{
${ }^{1}$ Disponível em: http://wiki.seeedstudio.com/Grove-GSR_Sensor/

${ }^{2}$ Disponível em: https://www.arduino.cc/en/Main/Arduino_BoardLeonardo
} 
positivas (i.e. usuário atinge um oitante com Valência positiva) quando interagindo na interface com as leis da Gestalt ocorreram em aproximadamente $65 \%$ das mudanças de estado emocional (44 casos de um total de 68); o restante, $35 \%$, o que representa 24 casos do total, refere-se à uma mudança negativa das emoções (i.e. atingiram ou permaneceram em valência negativa) em relação ao uso das leis.

Na Tabela 2 é possível visualisar e verificar que não houve grandes discrepâncias nos valores de quantidade de mudança para as duas diferentes ordens de interação aplicadas no conjunto de usuários. Assim, pode-se afirmar que a ordem de interação não causou impacto significativo nas mudanças observadas nos estados emocionais dos participantes. A ordem de interação é lida de cima para baixo: SG (Sem Gestalt) e G (Gestalt).

Tabela 2. Relação entre quantidade de mudanças e ordem das sessões.

\begin{tabular}{|c|c|c|c|c|c|c|c|c|c|c|c|c|c|c|c|}
\hline ID & 1 & 2 & 3 & 4 & 5 & 6 & 7 & 8 & 9 & 10 & 11 & 12 & 13 & 14 & 15 \\
\hline $\begin{array}{l}\text { Qtde. } \\
\text { Mudanças }\end{array}$ & 2 & 6 & 6 & 9 & 5 & 2 & 8 & 3 & 5 & 3 & 4 & 1 & 5 & 4 & 5 \\
\hline Ordem & $\mathrm{G}$ & $\mathrm{SG}$ & $\mathrm{SG}$ & $\mathrm{SG}$ & SG & $\mathrm{SG}$ & G & SG & SG & $\mathrm{G}$ & $G$ & SG & $\mathrm{G}$ & $\mathrm{G}$ & $\mathrm{SG}$ \\
\hline Versões & $\mathrm{SG}$ & $\mathrm{G}$ & G & $\mathrm{G}$ & G & $\mathrm{G}$ & SG & $\mathrm{G}$ & $\mathrm{G}$ & $\mathrm{SG}$ & $\mathrm{SG}$ & $\mathrm{G}$ & SG & $\mathrm{SG}$ & $\mathrm{G}$ \\
\hline
\end{tabular}

Ademais, com os octantes resultantes da classificação dos dados de cada voluntário, fez-se o cálculo da moda dos octantes mais presentes para as tarefas realizadas nas duas versões da solução web. Com isso, podemos averiguar de forma mais direta as variações emocionais significativas por tarefa. $\mathrm{O}$ símbolo $X$ significa que não foi possível identificar um valor para a moda para aquele sensor, pois não apresentou mudança no estado emocional.

Tabela 3. Oitantes predominantes das mudanças de emoções.

\begin{tabular}{|c|c|c|c|c|c|c|c|c|}
\hline \multicolumn{2}{|c|}{ Tarefa } & 1 & 2 & 3 & 4 & 5 & 6 & 7 \\
\hline \hline \multirow{4}{*}{ G } & GSR & 8 & 8 & 2 & 2 e 8 & 8 & 8 & 8 \\
\cline { 2 - 9 } & ECG & 7 & 2 & 7 & 7 & 2 & 7 & 7 \\
\cline { 2 - 9 } & EEG & 8 & 8 & 1 e 8 & 1 e 7 & $\times$ & 8 & 7 e 8 \\
\hline \hline \multirow{3}{*}{ SG } & GSR & 4 & 4 e 8 & 8 & 8 & 4 & 4 & 4 \\
\cline { 2 - 8 } & ECG & 2 & 7 & 7 & 7 & 7 & 2 & 2 \\
\cline { 2 - 8 } & EEG & 3 & 1 & 1 & 2 e 8 & $\times$ & 1 & 1 e 3 \\
\hline
\end{tabular}

$\mathrm{Na}$ Tabela 4 é exposta a relação percentual das mudanças emocionais por tarefa. Se o estado emocional de cada individuo (medido pelo oitante resultante), em uma dada tarefa $\mathrm{G}$, ficou diferente do estado emocional na mesma tarefa SG, computou-se como mudança. Não se julgou se a mudança foi positiva ou negativa. A média foi em torno de $54 \%$ de diferenças emocionais ao longo das 7 tarefas. Com destaque para as tarefas 3, 4 e 6 .

Entretanto, apesar da presença de mudanças, em algumas situações os resultados dos sensores entraram em conflito em relação à influência esperada das leis, que foram os casos dos usuários de IDs 3 e 13 em duas das sete tarefas. 
Tabela 4. Análise dos resultados das classificações dos sinais fisiológicos.

\begin{tabular}{|c|c|c|}
\hline Tarefa & Mudanças & Sem Alteração \\
\hline 1 & $40 \%$ & $60 \%$ \\
\hline 2 & $46 \%$ & $54 \%$ \\
\hline 3 & $66 \%$ & $34 \%$ \\
\hline 4 & $73 \%$ & $27 \%$ \\
\hline 5 & $40 \%$ & $60 \%$ \\
\hline 6 & $60 \%$ & $40 \%$ \\
\hline 7 & $53 \%$ & $47 \%$ \\
\hline
\end{tabular}

\section{Conclusão e Trabalhos Futuros}

Com os resultados finais, pôde-se verificar que um conjunto de leis da Teoria da Gestalt conseguiu influenciar as emoções de uma parcela do grupo de voluntários, sugerindo que a sua aplicação em elementos de uma interface web pode trazer uma melhora no estado emocional de alguns participantes. No entanto, ainda existem outras leis para serem estudadas e que podem ser úteis para futuras pesquisas e aplicações em interfaces de usuário para soluções web.

Por esta razão, para trabalhos futuros, seria necessário a coleta de dados com um grupo de usuários mais heterogêneo em idade, familiaridade com tecnologias, nível de escolaridade e área de atuação. Com relação às leis da Gestalt, pela falta da aplicação de algumas leis, pode ser desenvolvido uma nova interface web para implementá-las. Além disso, poderia-se ter a captação dos mesmos sinais fisiológicos e utilizar outras técnicas ou sistemas para uma classificação dos dados já obtidos ou desses novos dados.

\section{Referências}

Abadi, M. K. and et al. (2015). DECAF: meg-based multimodal database for decoding affective physiological responses. IEEE Transactions on Affective Computing, 6(3):209-222.

Beale, R. and Peter, C. (2008). The Role of Affect and Emotion. In Affect and Emotion in Human-Computer Interaction, volume 4868, pages 1-11.

Boring, E. G. (1942). Sensation and perception in the history of experimental psychology. Appleton Century Crofts, New York.

Chang, D. and Nesbitt, K. V. (2006). Identifying Commonly-Used Gestalt Principles as a Design Framework for Multi-Sensory Displays. In 2006 IEEE International Conference on Systems, Man and Cybernetics, volume 3, pages 2452-2457.

Chang, L. J. and et al. (2015). A Sensitive and Specific Neural Signature for PictureInduced Negative Affect. PLOS Biology, 13(6):1-28.

Cristescu, I. (2008). Emotions in Human-Computer Interaction: the role of nonverbal behavior in interactive systems. In Revista Informatica Economică, volume 12, pages 110-116.

Gonçalves, V. P. (2016). Uma abordagem para indicar o estado emocional de usuários em tempo de interaração. PhD thesis, Universidade de São Paulo. 
Koelstra, S. and et al. (2012). Deap: a database for emotion analysis; using physiological signals. IEEE Transactions on Affective Computing, 3(1):18-31.

Köhler, W. (1920). Physical Gestalten (Die Physischen Gestalten in Ruhe und im stationaren Zustand). Eine naturphilosophische Untersuchung, Erlangen. [W. D. Ellis (Ed.) A sourcebook of Gestaltpsychology. London: Routledge Kegan Paul, 1938, pp. 17-54].

Lichtenstein, A. and et al. (2008). Comparing Two Emotion Models for Deriving Affective States from Physiological Data, pages 35-50. Springer Berlin Heidelberg, Berlin, Heidelberg.

Mahlke, S. and Mingue, M. (2008). Consideration of multiple components of emotions in human-technology interaction. In Affect and emotion in human-computer interaction, pages 51-62. Springer.

Norman, D. A. (2004). Emotion design: why we love (or hate) everyday things.

Palmer, S. E. and Rock, I. (1992). Common region: a new principle of perceptual grouping. Cognitive Psychology, 24:436-447.

Palmer, S. E. and Rock, I. (1994). Rethinking perceptual organization: the roles of uniform connectedness. Psychonomic Bulletin and Review, 1:29-55.

Piccolo, S. L., Hayashi, E. C. S., and Baranauskas, M. C. C. (2010). Psicologia geral. $2^{o}$ Workshop sobre Aspectos da Interação Humano-Computador para a Web Social, pages 29-38.

Scherer, K. R. (1984). On the nature and function of emotion: a component process approach. Approaches to emotion, 2293:317.

Scherer, K. R. (2001). Appraisal considered as a process of multilevel sequential checking. Appraisal processes in emotion: Theory, methods, research, 92(120):57.

Scherer, K. R. (2005). What are emotions? And how can they be measured? Social science information, 44(4):695-729.

Shami, N. S. and et al. (2008). Measuring Affect in HCI: going beyond the individual. In Proceedings of ACM CHI 2008 Conference on Human Factors in Computing Systems, pages 3901-3904.

Souza, I. E. and Neris, V. P. A. (2019). Classificação de Sinais Fisiológicos para Inferência do Estado Emocional de Usuários. Master's thesis, Universidade Federal de São Carlos (UFSCar), São Carlos, São Paulo.

Subramanian, R. and et al. (2016). ASCERTAIN: emotion and personality recognition using commercial sensors. IEEE Transactions on Affective Computing, 9(2):147-160.

Wertherimer, M. (1924). Gestalt Theory (Uber Gestalttheorie). [W. D. Ellis (Ed.) A sourcebook of Gestalt psychology. London: Routledge Kegan Paul, 1938, pp. 1-11].

Xavier, R. A. C., Garcia, F. E., and Neris, V. P. A. (2012). Decisões de Design de Interfaces Ruins e o Impacto delas na Interação: um estudo preliminar considerando o estado emocional de idosos. pages 127-136. 